\title{
Cancer Stem Cells in Pediatric Brain Tumors
}

\author{
Miranda M. Tallman'1,2 • Abigail A. Zalenski ${ }^{1,3} \bullet$ Monica Venere $^{1}$ \\ ${ }^{1}$ Department of Radiation Oncology, James Cancer Hospital and Comprehensive Cancer \\ Center, The Ohio State University Wexner School of Medicine, Columbus, Ohio, USA; \\ ${ }^{2}$ Biomedical Sciences Graduate Program, The Ohio State University, Columbus, OH, USA; \\ ${ }^{3}$ Neuroscience Graduate Program, The Ohio State University, Columbus, OH, USA
}

Author for correspondence: Monica Venere, Department of Radiation Oncology, James

Cancer Hospital and Comprehensive Cancer Center, The Ohio State University Wexner

School of Medicine, Columbus, Ohio, USA. Email: monica.venere@osumc.edu

Doi: https://doi.org/10.36255/exonpublications.gliomas.2021.chapter3

\begin{abstract}
Cancer stem cells are a subpopulation of tumor cells that have the ability to self-renew, initiate tumors in model systems, and differentiate into noncancer stem cells. They are also resistant to current standard of care treatments, such as radiotherapy and chemotherapy. Due to these properties, cancer stem cells contribute to tumor progression and recurrence and need to be inclusively targeted with therapeutic paradigms used in the clinical setting. This chapter covers the most up-to-date published information on cancer stem cells in the context of pediatric brain tumors. The characteristics of pediatric brain tumor cancer stem cells, including resistance mechanisms and differential genetic regulation that allow for the stem like phenotype, are presented. The current research on cancer stem cells in medulloblastoma, ependymoma, diffuse intrinsic pontine glioma, and pediatric gliomas as well as potential approaches that are being developed to target cancer stem cells are highlighted. Challenges in targeting cancer stem cells in the pediatric patient population are also discussed.
\end{abstract}

Keywords: cancer stem cells; chemotherapy resistance; pediatric neuro-oncology; radio-resistance; tumor initiating cells

In: Gliomas. Debinski W (Editor). Exon Publications, Brisbane, Australia. ISBN: 978-0-6450017-4-7; Doi: https://doi.org/10.36255/exonpublications.gliomas.2021

Copyright: The Authors.

License: This open access article is licenced under Creative Commons Attribution-NonCommercial 4.0 International (CC BY-NC 4.0) https://creativecommons.org/licenses/by-nc/4.0/ 


\section{INTRODUCTION}

There are more than 28,000 children in the United States currently living with brain tumors (1). Pediatric brain tumors are the second most common cancer in children and the leading cause of cancer related deaths $(1,2)$. There are many different types of pediatric brain tumors, and they have varying treatment options and survival outcomes. Advances in successful treatments and surgical techniques have allowed tumors, such as medulloblastoma and low-grade gliomas, to achieve a 5 -year survival of about $75 \%(3,4)$. Meanwhile, survival of other pediatric brain tumors, such as diffuse intrinsic pontine glioma (DIPG), high-grade glioma, and ependymoma remain dismal. DIPG, also known as diffuse midline glioma, is one of the most deadly pediatric brain tumors with an overall survival of $30 \%$ a year after diagnosis, and less than $1 \%$ after 5 years (5). Current treatments for pediatric brain tumors generally include surgery, radiotherapy, and/or chemotherapy (4). Even with these treatments, many pediatric tumors still remain incurable. Tumors like ependymoma have poor prognosis due to high rates of recurrence, adding another layer of complexity to treatment planning for pediatric tumors.

One of the reasons these pediatric brain tumors are difficult to cure and commonly recur is the existence of innate intra-tumor heterogeneity. Using genomic sequencing and clustering algorithms, brain tumors have been shown to be heterogeneous in nature and to have distinct subpopulations existing within the same tumor. One leading theory as to how this high level of heterogeneity connects to a high recurrence rate and low survival is called the cancer stem cell (CSC) hypothesis (6-8).

The CSC hypothesis is based around the existence of a subpopulation of cells within a tumor that are able to initiate new tumors in in vivo model systems. These cells can both self-renew and differentiate as a means to repopulate tumors by producing both more CSCs as well as differentiated non-CSCs $(7,8)$. CSCs can be rare, in some cases making up less than 3\% of the cell population. However, if missed during surgery, the CSCs are capable of re-forming tumors, which results in tumor recurrence (7). CSCs found in brain tumors have also been shown to be resistant to aggressive radiotherapy and largely unaffected by standard chemotherapies, often leading them to be left behind after treatment (9). It has been shown CSCs will re-populate tumors and lead to resistant, aggressive secondary tumors (Figure 1) (9). Due to these aspects of CSCs, there exists a desperate need to not only further study this subpopulation in the context of pediatric brain tumors, but also to find therapeutics that target these cells to reduce recurrence.

In this chapter, we discuss the properties and features that make CSCs such a dynamic and important population to study, set in the context of their role in pediatric brain tumors. We first cover defining characteristics of CSCs, such as tumor initiation, self-renewal, and chemotherapy/radiation-resistant properties. Then we present different types of pediatric brain tumors that have known CSC subpopulations, and what work has been done to understand them. Lastly, we explore different ways researchers are targeting pediatric brain tumor CSCs. 


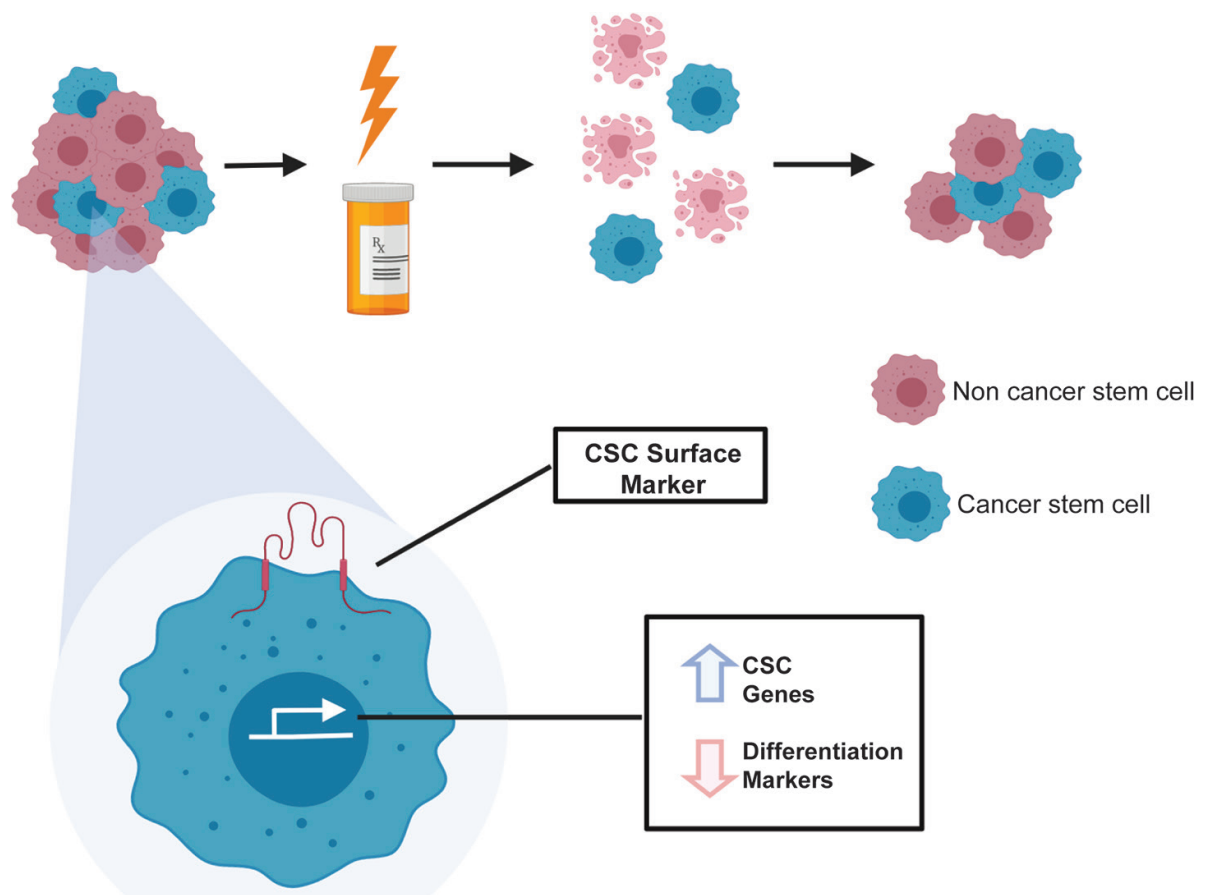

Figure 1. Pediatric brain tumor CSC phenotypes and genotypes. Pediatric brain tumors are heterogeneous in nature, containing both CSCs and non-CSCs. When bulk tumors are treated, with either radiotherapy or chemotherapies, the radio- and chemo-resistant CSC subpopulation remains and can repopulate the tumor. CSCs can be sorted from bulk tumors by cell surface markers, such as CD133. CSCs have intrinsic characteristics such as gene regulation that keep them in a progenitor/stem state via upregulation of stem genes and downregulation of genes related to a more differentiated cell state. These changes give CSCs the ability to self-renew and initiate tumor formation, thereby contributing to tumor recurrence. Differential gene regulation from non-CSCs is also thought to contribute to CSC therapeutic resistance, with DNA damage repair genes and drug efflux transporters upregulated, while pro-apoptotic pathways are downregulated. Created with BioRender.com.

\section{PEDIATRIC BRAIN TUMOR CSC PROPERTIES}

CSCs were first identified in acute myeloid leukemia (AML), where researchers discovered a subpopulation of tumor cells that were able to proliferate, differentiate, and self-renew, as shown by serial transplantation in mouse models $(10,11)$. Later, CSCs were found in numerous solid tumors, such as breast, prostate, colon, lung, and brain (12-17). CSCs have been identified in a variety of brain tumors, both adult and pediatric, such as glioblastoma, ependymoma, and medulloblastoma. Common practice for isolating CSCs is to first dissociate a bulk tumor into a single cell suspension, sort for the CSCs via reported cell-surface markers using fluorescence-activated cell sorting (FACS) or magnetic sorting, and then 
functionally validate the self-renewal capabilities, as well as the ability to accurately recapitulate the tumor of origin in orthotopic xenotransplantation studies in mice (9). The most common CSC cell-surface marker used for brain tumors is the extracellular glycosylated antigen known as CD133 (18). Validation of the CSC phenotype can be done through in vitro limiting dilution assays, in which lower and lower cell numbers are plated per well of a tissue culture plate down to one cell per well. The cells are given time to expand with the final metric evaluated being the presence or absence of a tumorsphere in each well. This can be quantified using the eLDA calculator, which is an open access online algorithm that calculates the frequency of CSCs present in a sample (19). Most important for functional validation, however, is an in vivo orthotopic limiting dilution assay where CSCs and matched non-CSCs are implanted intracranially in lower and lower cell numbers and the ability to initiate tumor formation is evaluated. CSCs alone are able to form a tumor that recapitulates the characteristics of the original patient tumor whereas the non-CSCs are unable to form tumors in mouse models. This ability to initiate tumors is a hallmark of functionally defining CSCs. Ependymoma, medulloblastoma, pediatric glioblastoma, and DIPG are some of the pediatric brain tumors in which CSC populations have been isolated and functionally validated via the in vivo functional assay (20-25).

\section{CSC treatment resistance}

As well as being able to re-form tumors in vivo, CSCs have also been shown to be resistant to irradiation and chemotherapy in many different tumor types. This evidence further supports the hypothesis that these cells contribute to tumor recurrence, since resistant CSCs can persist after administration of conventional treatments. In fact, many therapeutics have shown efficacy for non-CSCs, while having little to no efficacy for CSCs (9). In certain brain tumors, CSCs have been shown to be resistant to both radiation and chemotherapy due to their inherent cellular properties. One example is glioblastoma CSCs having an enhanced DNA damage response $(9,26-28)$. This means that when DNA in glioblastoma CSCs is damaged by either radiation or chemotherapy, they are better able to repair the damage and survive than non-CSCs that die as a result of the increased DNA damage. It has been shown that CSCs in adult gliomas have an increased DNA damage repair capability via the ATR/Chk1 and ATM/Chk2 pathways, enhanced reactive oxygen species (ROS) scavenging, which protects against damage induced by radiation, and activation of cell survival pathways like PI3K/AKT $(26,29,30)$. For pediatric gliomas, ependymoma, and medulloblastoma, CSCs have been shown to be resistant to many DNA damaging chemotherapeutics. This increased DNA damage response (DDR) is thought to be due to many factors discussed above, as well as other molecular aspects such as downregulation of apoptotic pathways, upregulation of anti-apoptotic pathways, and upregulation of pro-survival pathways (22). In general, CSCs are also thought to achieve resistance through slower replication rates, increased drug efflux, alterations of cell death pathways, and alterations in drug metabolism (31).

An example of upregulated drug efflux pumps in CSCs is the ABC transporter family, which has been attributed to CSC resistance mechanisms. ABC transporters have been shown to play a role in drug resistance in many cancer types, due 
to their ability to efflux cytotoxic drugs and maintain drug concentrations inside cells at sub-therapeutic levels $(29,32)$. Temozolomide and mitoxantrone are some of many drugs glioma CSCs have been shown to be resistant to, partly due to $\mathrm{ABC}$ transporters (33). In fact, temozolomide has been shown to increase the fraction of adult glioma CSCs in treated tumorspheres (33). ABC transporters are thought to be able to pump the chemotherapies out of the CSCs, leading to cell survival. $\mathrm{ABC}$ transporters have been found to be overexpressed in CSCs, and their presence correlates with high levels of drug resistance $(29,33,34)$.

Another potential resistance mechanism described in brain tumor CSCs is increased aldehyde dehydrogenase (ALDH) activity. ALDH proteins play a role in the maintenance of CSCs and have been shown to be a marker of radio-resistance in gliomas. These also correlate with clonogenicity, or self-renewal ability, as well as tumor initiation in vivo (29). The ALDH1 and ALDH3Al proteins are thought to play a role in resistance to radiation through the reduction of free radicals in the cells caused by radiation therapy, through the production of NAD(P)H (29).

\section{CSC genetic changes in pediatric brain tumors}

CSCs have been shown to have many genetic and phenotypic differences when compared to non-CSCs. Investigations are ongoing to understand the overall genetic makeup of CSCs, such as the changes in CSCs that allow for increased resistance to conventional treatments. While a majority of what we know about genetic and epigenetic changes in CSCs comes from adult tumor research, this has been explored in some pediatric brain tumors as well. CSCs overexpress a group of genes that allow them to exhibit stem cell characteristics. One example is medulloblastoma in which several molecular signaling pathways are upregulated in CSCs, including Sonic Hedgehog (SHH), MYC proto-oncogene, and Notch. SHH is a pathway that promotes progenitor proliferation, and over-activation is linked with tumorigenesis (22). Indeed, SHH target genes, Gli1 and CyclinD1I, are overexpressed in medulloblastoma CSCs. CSC tumorsphere self-renewal capabilities were also shown to be dependent on N-MYC, and were associated with medulloblastoma CSC stemness (22). Notch signaling was found to be important in the regulation of CSCs by maintaining them in an undifferentiated state (22). For example, DIPG exhibits amplification of ERBB1 and mutations in the TP53 gene. In childhood gliomas, mutations in $H 2 F 2 A$ and DAZZ were found. Current work is being performed to uncover how these genetic changes promote CSCs stemness in their respective tumors (9). In ependymoma, CSCs have been found to have gene expression profiles very similar to radial glia cells (RGCs), a type of progenitor cell found in the wall of neural tubes during the earliest stages of development, which play an important role in neuronal fate $(20,35)$. CD133, Nestin, RC2 and BLBP were all shown to be overexpressed in ependymoma CSCs as well as RGCs (20). Ependymoma CSCs grown in a subcutaneous xenograft model also show a high degree of similarity with adult neural stem cells (NSCs) (21). Messenger RNA (mRNA) expression for genes such as EPHB-EPHRIN and Notch are similar between the two and both play a role in maintaining stem cell states $(20,21)$. This highlights a key property of brain tumor CSCs: they genetically have significant overlap with progenitors and stem cells native to the brain. This is true for both pediatric and adult brain tumor CSCs, as adult glioblastoma CSCs harbor transcriptional programs akin to progenitors as well $(36,37)$. 
In summary, pediatric and adult CSCs harbor genetic upregulation of stem cell genes, allowing them to stay in a pluripotent state. Resistance has been linked to this stem fate, so research has been done in adult glioblastoma CSCs to push the stem cells to a more differentiated, and therefore more sensitive fate $(38,39)$. PARP inhibitors, high-Z metal nanoparticles, PI3K inhibitors, DNA repair inhibitors, pimozide, CBL0137, various microRNAs, and many other therapeutic techniques have been shown to sensitize glioblastoma to irradiation (39-43). PARP inhibitors have been shown in glioblastoma to decrease the CSC frequency in vivo, leading to a higher percentage of non-CSCs in the tumor. They also led to a significant increase overall survival in murine models when PARP inhibitors were combined with radiotherapy, compared to either therapy alone (40). CBL0137, an anticancer drug that targets the FACT complex, has been shown to increase asymmetric cell division in glioblastoma CSCs, resulting in an increase in non-CSCs (38). The inhibition of FACT in glioblastoma CSCs resulted in downregulation of SOX2, OCT4, NANO, OLIG2, and NES on the mRNA level (38). Together, these data highlight the importance of understanding the genetic drivers behind CSCs and potential ways researchers can develop therapeutics to target these genetic differences.

\section{CSCS ACROSS PEDIATRIC BRAIN TUMOR TYPES}

There are many overlapping characteristics between all pediatric brain tumor CSCs, such as tumor initiation, increased resistance mechanisms, as well as stem genes upregulated, resulting in an undifferentiated phenotype. Despite the similarities, medulloblastoma, ependymoma, DIPG, and gliomas all contain genetically distinct CSC subpopulations, specific to each tumor type (Table 1). The following sections explore what is known about the CSC population in each of these cancers.

\section{Medulloblastoma CSCs}

Medulloblastoma is the most common pediatric brain tumor. Medulloblastoma was shown to have intratumor heterogeneity, aiding the discovery of CSCs with unlimited self-renewal, increased invasion and motility, and thus the ability to contribute to tumor recurrence (22). CD133-positive medulloblastoma tumor cells have been shown to be able to self-renew in vitro (22). In vivo, when as little as 100 CD133-positive cells were intracranially injected into an immunodeficient mouse, the resultant tumor resembled the original medulloblastoma patient tumor, whereas CD133-negative cells, regardless of number injected, did not form a tumor $(22,44)$. It has also been shown that Nestin overexpressing progenitor-like cells in transgenic mouse models develop gliomas and medulloblastomas, showing Nestin as another CSC marker in medulloblastoma (45). Separately, it has been shown that patients with tumors displaying prominent CSC signatures correlate with poor prognosis (22).

Medulloblastoma has been described to have four subtypes, WNT, SHH, Group 3 and Group 4, with the latter two being the most aggressive (46-49). CSCs have been identified in Group 3, and were demonstrated to be able to form 


\section{TABLE 1 Summary of published findings on CSCs in pediatric brain tumors}

\begin{tabular}{|c|c|c|c|c|}
\hline Tumor Type & Description & Markers and Genes & $\begin{array}{l}\text { Example } \\
\text { Therapeutics } \\
\text { Targeting CSCs }\end{array}$ & References \\
\hline Medulloblastoma & $\begin{array}{l}\text { Increased invasion } \\
\text { and motility, high } \\
\text { recurrence, } 100 \text { CSCs } \\
\text { enough to form tumor } \\
\text { in mice, self-renewal }\end{array}$ & $\begin{array}{l}\text { CD133 positive } \\
\text { CD15 positive } \\
\text { Sox2 positive } \\
\text { high Nestin expression } \\
\text { high MYC expression }\end{array}$ & $\begin{array}{l}\text { Smo-agonist } \\
\text { (to inhibit } \\
\text { SHH } \\
\text { pathway), } \\
\text { vismodegib, } \\
\text { PI3K/Akt } \\
\text { inhibitors }\end{array}$ & $22,44,45,50$ \\
\hline Ependymoma & $\begin{array}{l}\text { Tumor initiation, self- } \\
\text { renewal, resistance } \\
\text { to conventional } \\
\text { chemotherapies }\end{array}$ & $\begin{array}{l}\text { CD133 positive } \\
\text { high Nestin expression } \\
\text { RC2 positive }\end{array}$ & $\begin{array}{l}\text { Temozolomide, } \\
\text { VP16, } \\
\text { vincristine, } \\
\text { cisplatin, } \\
\text { vorinostat }\end{array}$ & $20,21,51$ \\
\hline DIPG & $\begin{array}{l}\text { Self-renewal, tumor } \\
\text { initiation, small } \\
\text { fraction of cells within } \\
\text { the tumor }\end{array}$ & $\begin{array}{l}\text { Ki- } 67 \text { positive } \\
\text { Olig2 positive } \\
\text { Nestin positive } \\
\text { GFAP positive } \\
\text { PDGFR } \alpha \text { positive }\end{array}$ & & $23,24,52,53$ \\
\hline Glioma & $\begin{array}{l}\text { Resistant to radiation, } \\
\text { resistant to } \\
\text { chemotherapy, tumor } \\
\text { initiation, leads to } \\
\text { recurrence, pediatric } \\
\text { glioblastoma was } \\
\text { able to recapitulate } \\
\text { tumor (antigenic with } \\
\text { hemorrhagic areas, } \\
\text { highly proliferative, } \\
\text { highly vasculature, } \\
\text { necrotic core) }\end{array}$ & $\begin{array}{l}\text { CD133 positive } \\
\text { High VEGF expression }\end{array}$ & $\begin{array}{l}\text { Oncolytic } \\
\text { herpesvirus, } \\
\text { anti-VEGF, } \\
\text { bevacizumab }\end{array}$ & $25,54,55$ \\
\hline
\end{tabular}

neurospheres in culture, with upregulation of CD133, Nestin, and Musashi, which are all stem cell gene markers (50). These medulloblastoma CSCs were able to propagate tumor formation in mice, and able to recapitulate the primary tumor (50). Group 3 medulloblastoma also includes MYC amplification and overexpression, which is essential for medulloblastoma stem cell initiation and self-renewal properties, and correlates with the aggressiveness of the stem cells in these tumors (22). These cells also display therapeutic resistance in the form of quiescence, regulation of key pathways (downregulation of apoptotic pathways and upregulation of pro-survival pathways), and manipulation of microenvironmental factors (hypoxia) $(22,50)$. Additional research is needed to investigate the other subtypes and any CSCs they main contain. 


\section{Ependymoma CSCs}

Ependymoma tumors are found primarily in children but can occur in patients of all ages. Ependymomas are generally found in the spine in adult patients and have a good prognosis, while pediatric patients tend to have intracranial ependymomas that correlate with poor outcomes. A distinct CSC subpopulation has been identified in intracranial ependymomas. These ependymoma CSCs have also been shown to be capable of tumor initiation, and are also resistant to conventional chemotherapies (21). Ependymoma CSCs have been able to recapitulate the original tumor when orthotopically implanted into a mouse $(20,51)$. Several studies have been done in order to target these resistant populations. The anticancer drugs temozolomide (alkylating agent), VP16 (topoisomerase II inhibitor), vincristine (vinca alkaloid), cisplatin (alkylating agent), and vorinostat (an HDAC inhibitor) have all been used to target ependymoma CSCs with varying degrees of success $(21,51)$. In some studies, temozolomide and vorinostat both decreased ependymoma CSC viability, while another study has shown ependymoma CSCs are resistant to temozolomide, VP16, vincristine, and cisplatin $(21,51)$. Thus, a reliable way to target these CSCs remains elusive.

\section{DIPG CSCs}

DIPG is an incurable pediatric brain tumor with less than a $1 \% 5$-year overall survival rate and has one of the worse prognosis of any pediatric brain tumor with a mean age of 6-7 years old at diagnosis (52). DIPG CSCs have not been studied in depth, but it has been found that primary cell lines from DIPG patients had a population of CSCs that could self-renew in vitro and form tumors in immunodeficient mice in vivo $(23,53)$. In another study, DIPG was shown to contain a subpopulation of cells highly expressing stem cell genes (24). DIPG CSCs are a genetically distinct subpopulation defined by single cell RNA-seq and were found to also be genetically distinct from other glioma CSCs (24). DIGP tumor cells grown in stem promoting media have tumor-initiation capacity. Although not quantified, it was demonstrated that the CSCs in DIPG were a minority, only making up a small fraction of the total tumor. The total tumor population had a differentiated signature, similar to that described within the CSC hypothesis (24).

\section{Gliomas CSCs and other pediatric brain tumors}

High-grade gliomas (HGG), which include pediatric glioblastoma, represent about $10 \%$ of pediatric brain tumors. Pediatric glioblastoma has high morbidity and mortality and about a 20\% overall survival rate, and this outcome can be in part attributed to glioma CSCs (25). The CSCs have been shown to be resistant to radiation and chemotherapy and to have the ability to repopulate the tumor, causing recurrence after initial treatment in in vivo model systems (25). Glioma CSCs have also been shown to able to accurately recreate HGG tumors in these model systems, in terms of epigenetic post-translational modifications, copy number alterations, and DNA mutations (54). The CSCs also formed neurospheres in vitro, validating self-renewal properties (54). 
In one specific example, a pediatric glioblastoma from a 4 year old patient was sorted for CD133-positive cells which were then injected orthotopically into immunocompromised mice (55). The CSCs formed tumors that displayed highly antigenic and hemorrhagic areas and had areas that were highly proliferative, with increased vasculature and necrosis (55). It was identified that pediatric glioblastoma CSCs had elevated levels of vascular endothelial growth factor (VEGF), a signaling protein known for its role in angiogenesis (55). The level of VEGF was 10- to 20-fold higher in CSCs compared to the non-CSCs, highlighting a potential way to target the CSCs in vivo (55).

\section{TARGETING PEDIATRIC CSC}

Targeting pediatric CSCs will be pivotal for eradicating tumors completely and reducing recurrence. Medulloblastoma CSCs have been targeted in a number of ways. Inhibiting Notch signaling has been shown to reduce CD133-positive cell count almost 5-fold, while also increasing apoptotic rates 10-fold (9). Inhibition of the SHH pathway via Smo-antagonist reduced Nanog expression and inhibited the self-renewal ability of the medulloblastoma CSCs (22). The SHH pathway has also been shown to play a key role in medulloblastoma CSC self-renewal and maintenance via Bmi-1 promotion of CSC tumorigenicity (22). However, targeting this pathway with vismodegib led to increased CSC quiescence instead of leading to cell death (22). Targeting another pathway, PI3K/Akt in a mouse model of medulloblastoma, led to radio-sensitization of CSCs and an increase in apoptosis $(22,33)$.

As mentioned earlier, ependymoma CSCs have been shown to be sensitive to vorinostat, and to some degree to temozolomide. It was shown that vorinostat decreases tumorsphere-initiating capacity and induced differentiation. Ability to initiate tumors, or form tumorspheres in vitro, is a hallmark of CSCs, so the ability of vorinostat to stop the formation of these spheres shows potential for targeting ependymoma CSCs (21). Temozolomide was shown to have no effect on ependymoma CSCs, however in another study, temozolomide was found to decrease tumor initiation, moderately increase survival in vivo in an intracranial mouse model, and decrease subcutaneous tumor volume in combination with VP16 $(21,51)$. Much more remains to be done to find a reliable treatment for pediatric CSCs.

There have been many creative ways developed to target CSCs. One proposed approach is via an oncolytic herpesvirus, which has been used to target HGG CSCs. The premise is to target and kill the CSCs and non-CSCs, while sparing normal brain tissue (25). Pre-clinical studies have shown engineered oncolytic herpes simplex virus can infiltrate, replicate within and then lyse HGG CSCs, leading to prolonged survival in in vivo mouse models intracranially injected with gliomas (25). Retinoic acid has also been shown to differentiate glioma CSCs. However, this has not been uniformly seen in all gliomas (56). Bevacizumab, an anti-angiogenic drug targeting VEGF, has been used to treat pediatric glioblastoma CSCs and has demonstrated suppressed growth in xenograft models (55). Bevacizumab is used now in combination with the current standard of care, temozolomide after surgery and radiotherapy, in clinical trials (ACNS0822) (25). 


\section{Current challenges in treating pediatric brain tumors}

One of the most complicated factors in treating pediatric brain tumors is preserving the developing brain while simultaneously eradicating the malignant tumor cells. Since CSCs and normal neural stem cells (NSCs) and neural progenitor cells (NPCs) have many overlapping characteristics, phenotypes, and genetic expression, there is potential to damage the normal developing brain tissue during tumor treatment (11). Therapeutics need to be developed with this in mind, in a way that targets CSCs and not normal NSCs/NPCs which are necessary and critical to the developing brain. Treatments for pediatric neuro-oncology in general must be particularly non-toxic to the vulnerable young brain, with a number of therapies potentially leading to irreversible damage, loss of cognition, and stunting brain development. Targeting tumorigenic pathways, specific growth factors, and microenvironmental factors that play a role in CSC plasticity are the suggested ways of specifically targeting CSCs (9).

Current treatment for pediatric brain tumors is surgery, irradiation, and/or chemotherapy. Chemotherapy is occasionally used in very young children in an attempt to stall tumor growth until they are of an age where they can tolerate more aggressive treatments like radiotherapy. However, chemotherapy also has drawbacks, some of which include early growth failure, nausea, cachexia, and deficiency in nutritional uptake (57). These effects are long term, as even replacement hormonal therapies cannot completely compensate for growth issues (58-62). Neurological defects leading to long term health issues, including major visual defects, severe hearing loss, and trouble with schooling, have been shown in patients given radiation treatment when less than 4 years old, $(63,64)$. One study found that only 1 in 3 long-term survivors of pediatric medulloblastoma were able to live completely normal lives, with the other $66 \%$ unable to be employed and reliant on social support network (63). These effects are thought to be a direct result of irradiation of the developing brain. Recent advances in radiotherapy, such as proton therapy, offer much better options for minimizing collateral damage to the brain. However, there is still a desperate need for new therapeutic interventions that would either allow for the postponement or replacement of irradiation in treating pediatric brain tumors, and specific targeting of CSCs that would not affect NSCs/NPCs.

While these are daunting hurdles, progress is being made to better treat pediatric patients. Firstly, understanding the biology and research from basic science to clinical trials is underway in order to create better treatments. Multidisciplinary collaborations between researchers aim to improve patient treatments via the better design of clinical trials $(65,66)$. Another step forward is identifying pediatric patients whose tumor allows for less intensive treatment. If less chemotherapy and/or radiation can still be used to cure the tumor, this will result in lessening of unnecessary side effects (66). Medulloblastoma is an example of these concepts. Basic science research studying medulloblastoma identified the WNT subtype and correlated it was a positive prognostic outcome. There are ongoing clinical trials for reducing radiotherapy in patients with the WNT subtype (66). 


\section{CONCLUSION}

Pediatric brain tumors are a serious, life altering occurrence in which multiple factors need to be balanced for optimal treatment, including protecting the developing brain, while still aggressively targeting the malignant tumor cells. Pediatric brain tumors have been shown to have intra-tumor cell heterogeneity, with a small population of CSCs present. So far, CSCs have been identified in gliomas, DIPG, ependymoma, and medulloblastoma. These cells have been shown to be capable of self-renewal and tumor initiation, two major hallmarks of CSCs. CSCs are also resistant to radiotherapy and many different chemotherapies, leading to the hypothesis that they play a key role in tumor recurrence and ultimately contribute to the high morbidity of these tumors. New ways of treating these tumors that inclusively target the CSC subpopulation are essential. Work has been done to specifically eradicate these CSCs therapeutically by inhibiting key pathways or targeting unique characteristics. Moving forward, monotherapies or combination treatments that treat all subpopulations of cells within the tumors, including CSCs, will result in prolonged survival and overall better outcomes for patients.

Conflict of interest: The authors declare no potential conflict of interest with respect to research, authorship, and/or publication of this chapter.

Copyright and permission statement: The authors confirm that the materials included in this chapter do not violate copyright laws. Where relevant, appropriate permissions have been obtained from the original copyright holder(s), and all original sources have been appropriately acknowledged or referenced.

\section{REFERENCES}

1. Brain Tumor Education: American Brain Tumor Association.https://www.abta.org/about-brain-tumors/ brain-tumor-education/ [Accessed on 18 Nov 2020]

2. Pollack IF. Brain tumors in children. N Engl J Med. 1994;331:1500-7. https://doi.org/10.1056/ NEJM199412013312207

3. Pollack IF, Agnihotri S, Broniscer A. Childhood brain tumors: current management, biological insights, and future directions. J Neurosurg Pediatr. 2019;3:261-73. https://doi.org/10.3171/2018.10. PEDS18377

4. Segal D, Karajannis MA. Pediatric Brain Tumors: An Update. Curr Probl Pediatr Adolesc Health Care. 20161;46(7):242-250. https://doi.org/10.1016/j.cppeds.2016.04.004

5. Mathew RK, Rutka JT. Diffuse Intrinsic Pontine Glioma : Clinical Features, Molecular Genetics, and Novel Targeted Therapeutics. J Korean Neurosurg. 2018;3:343-51. https://doi.org/10.3340/ jkns.2018.0008

6. Reya T, Morrison SJ, Clarke MF, Weissman IL. Stem cells, cancer, and cancer stem cells. Nature 2001;414(6859):105-11. https://doi.org/10.1038/35102167

7. Rich JN, Bao S. Chemotherapy and Cancer Stem Cells. Cell Stem Cell. 2007;1(4):353-5. https://doi. org/10.1016/j.stem.2007.09.011

8. Visvader JE, Lindeman GJ. Cancer stem cells in solid tumours: accumulating evidence and unresolved questions. Nat Rev Cancer. 2008;8(10):755-68. https://doi.org/10.1038/nrc2499

9. Tamara J. Abou-Antoun JSH, Justin D. Lathia, Stephen M. Dombrowski. Brain Cancer Stem Cells in Adults and Children: Cell Biology and Therapeutic Implications. Neurotherapeutics. 2017;14:372-84. https://doi.org/10.1007/s13311-017-0524-0 
10. Bonnet D, Dick JE. Human acute myeloid leukemia is organized as a hierarchy that originates from a primitive hematopoietic cell. Nat Med. 1997;3:730-7. https://doi.org/10.1038/nm0797-730

11. Castelo-Branco P, Tabori U. Promises and challenges of exhausting pediatric neural cancer stem cells. Pediatr Res. 2012;71:523-8. https://doi.org/10.1038/pr.2011.63

12. Al-Hajj M, Wicha MS, Benito-Hernandez A, Morrison SJ, Clarke MF. Prospective identification of tumorigenic breast cancer cells. PNAS. 2003;100:3983-8. https://doi.org/10.1073/pnas.0530291100

13. Collins AT, Berry PA, Hyde C, Stower MJ, Maitland NJ. Prospective Identification of Tumorigenic Prostate Cancer Stem Cells. Cancer Res. 2005;65:10946-51. https://doi.org/10.1158/0008-5472. CAN-05-2018

14. Ricci-Vitiani L, G LD, Pilozzi E, Biffoni M, Todaro M, Peschle C, et al. Identification and expansion of human colon-cancer-initating cells. Nature. 2006;445:111-5. https://doi.org/10.1038/nature05384

15. Ho MM, Ng AV, Lam S, Hung JY. Side population in human lung cancer cell lines and tumors is enriched with stem-like cancer cells. Cancer Res. 2007;67:4827-33. https://doi.org/10.1158/00085472.CAN-06-3557

16. Eramo A, Lotti F, Sette G, Pilozzi E, Biffoni M, Di Virgilio A, et al. Identification and expansion of the tumorigenic lung cancer stem cell population. Cell Death Differ. 2008:504-14. https://doi. org/10.1038/sj.cdd.4402283

17. Singh SK, Clarke ID, Terasaki M, Bonn VE, Hawkins C, Squire J, et al. Identification of a Cancer Stem Cell in Human Brain Tumors. Cancer Res. 2003;63:5821-8.

18. Glumac PM, LeBeau AM. The role of CD133 in cancer: a concise review. Clin Transl Med. 2018. https://doi.org/10.1186/s40169-018-0198-1

19. Hu Y, Smyth G. ELDA: extreme limiting dilution analysis for comparing depleted and enriched populations in stem cell and other assays. J Immunol Methods. 2009;347. https://doi.org/10.1016/j. jim.2009.06.008

20. Taylor MD, Poppleton H, Fuller C, Su X, Liu Y, Jensen P, et al. Radial glial cells are candidate stem cells of ependymoma. Cancer Cell. 2005;8:323-35. https://doi.org/10.1016/j.ccr.2005.09.001

21. Milde T, Kleber S, Korshunov A, Witt H, Hielscher T, Koch P, et al. A novel human high-risk ependymoma stem cell model reveals the differentiation inducing potential of the histone deacetylase inhibitor Vorinostat. Acta Neuropathol. 2011:637-50. https://doi.org/10.1007/s00401-011-0866-3

22. Huang G-H, Xu Q-F, Cui Y-H, Li N, Bian X-W, Lv S-Q. Medulloblastoma stem cells: Promising targets in medulloblastoma therapy. Cancer Sci. 2016;107:583-9. https://doi.org/10.1111/cas.12925

23. Sun Y, Xu C, Pan C, Chen X, Geng Y, Wu Y, et al. Diffuse Intrinsic Pontine Gliomas Exhibit Cell Biological and Molecular Signatures of Fetal Hindbrain-Derived Neural Progenitor Cells. Neurosci Bull. 2019:216-24. https://doi.org/10.1007/s12264-018-00329-6

24. Filbin MG, Tirosh I, Hovestadt V, Shaw M, Escalante LE, Mathewson ND, et al. Developmental and oncogenic programs in H3K27M gliomas dissected by single-cell RNA-seq. Cancer Genomics. Science. 2018;360(6386): 331-5. https://doi.org/10.1126/science.aao4750

25. Friedman GK, Raborn J, Kelly VM, Cassady KA, Markert JM, Gillespie Y. Pediatric glioma stem cells: biologic strategies for oncolytic HSV virotherapy. Front Oncol. 2013;3. https://doi.org/10.3389/ fonc. 2013.00028

26. Bao S, Wu Q, McLendon RE, Hao Y, Shi Q, Hjelmeland AB, et al. Glioma stem cells promote radioresistance by preferential activation of the DNA damage response. Nature. 2006;444:756-60. https:// doi.org/10.1038/nature05236

27. Fukumoto. Radiosensitization of cancer stem cells in glioblastoma by the simultaneous inhibition of parallel DNA damage response pathways. Ann Transl Med. 2017;75:4416-28. https://doi. org/10.21037/atm.2017.03.39

28. Carruthers RD, Ahmed SU, Ramachandran S, Strathdee K, Kurian KM, Hedley A, et al. Replication stress drives constitutive activation of the DNA damage response and radioresistance in glioblastoma stem-like cells. Cancer Res. 2018;78:5060-71. https://doi.org/10.1158/0008-5472.CAN-18-0569

29. Cojoc M, Mäbert K, Muders MH, Dubrovska A. A role for cancer stem cells in therapy resistance: Cellular andmolecular mechanisms. Semin Cancer Biol. 2015;31:116-27. https://doi.org/10.1016/j. semcancer.2014.06.004

30. Peitzsch C, Kurth I, Kunz-Schughart L, Baumann M, Dubrovska A. Discovery of the cancer stem cell related determinants of radioresistance. Radiother Oncol. 2013;108:378-87. https://doi. org/10.1016/j.radonc.2013.06.003 
31. Renovanz M, Kim E. Intratumoral heterogeneity, its contribution to therapy resistance and methodological caveats to assessment. Front Oncol. 2014;4. https://doi.org/10.3389/fonc.2014.00142

32. Leonard GD, Fojo T, Bates SE. The Role of ABC Transporters in Clinical Practice. Oncologist. 2003;8(5). https://doi.org/10.1634/theoncologist.8-5-411

33. Bleau A-M, Hambardzumyan D, Ozawa T, Fomchenko EI, Huse JT, Brennan CW, et al. PTEN/PI3K/ Akt pathway regulates the side population phenotype and ABCG2 activity in glioma tumor stem-like cells. Cell Stem Cell. 2009;4:226-35. https://doi.org/10.1016/j.stem.2009.01.007

34. Patrawala L, Calhoun T, Schneider-Broussard R, Zhou J, Claypool K, Tang DG. Side population is enriched in tumorigenic, stem-like cancer cells, whereas ABCG2+ and ABCG2- cancer cells are similarly tumorigenic. Cancer Res. 2005;65(14):6207-19. https://doi.org/10.1158/0008-5472.CAN-05-0592

35. Howard BM, Mo Z, Filipovic R, Moore AR, Antic SD, Zecevic N. Radial Glia Cells in the Developing Human Brain. Neuroscientist. 2009;14:459-73. https://doi.org/10.1177/1073858407313512

36. Galli R, Binda E, Orfanelli U, Cipelletti B, Gritti A, De Vitis S, et al. Isolation and Characterization of Tumorigenic, Stem-like Neural Precursors from Human Glioblastoma. Cancer Res. 2004;64(19); 7011-21. https://doi.org/10.1158/0008-5472.CAN-04-1364

37. Huang Z, Cheng L, Guryanova OA, Wu Q, Bao S. Cancer stem cells in glioblastoma - molecular signaling and therapeutic targeting. Protein Cell. 2010;1:636-55. https://doi.org/10.1007/ s13238-010-0078-y

38. Kam J, Dermawan T, Hitomi M, Silver DJ, Wu Q, Sandlesh P, et al. Pharmacological Targeting of the Histone Chaperone Complex FACT Preferentially Eliminates Glioblastoma Stem Cells and Prolongs Survival in Preclinical Models. Cancer Res. 2016:2432-42. https://doi.org/10.1158/0008-5472. CAN-15-2162

39. Tallman MM, Zalenski AA, Deighen AM, Schrock MS, Mortach S, Grubb TM, et al. The small molecule drug CBL0137 increases the level of DNA damage and the efficacy of radiotherapy for glioblastoma. Cancer Lett. 2021;499:232-42. https://doi.org/10.1016/j.canlet.2020.11.027

40. Venere M, Hamerlik P, Wu Q, Rasmussen RD, Song LA, Vasanji A, et al. Therapeutic targeting of constitutive PARP activation compromises stem cell phenotype and survival of glioblastoma-initiating cells. Cell Death Differ. 2013:258-69. https://doi.org/10.1038/cdd.2013.136

41. Bahreyni-Toossi M-T, Dolat E, Khanbabaei H, Zafari N, Azimian H. microRNAs: Potential glioblastoma radiosensitizer by targeting radiation-related molecular pathways. Mutat Res. 2019;816-8(111679). https://doi.org/10.1016/j.mrfmmm.2019.111679

42. Rath BH, Camphausen K, Tofilon PJ. Glioblastoma radiosensitization by pimozide. Translational Cancer Res. 2016:S1029-S32. https://doi.org/10.21037/tcr.2016.11.17

43. Choi J, Kim G, Cho SB, Im H-J. Radiosensitizing high-Z metal nanoparticles for enhanced radiotherapy of glioblastoma multiforme. J Nanobiotechnology. 2020;18(122). https://doi.org/10.1186/ s12951-020-00684-5

44. Singh SK, Hawkins C, Clarke ID, Squire JA, Bayani J, Hide T, et al. Identification of human brain tumour initiating cells. Nature. 2004;432:396-401. https://doi.org/10.1038/nature03128

45. Nakano I, Kornblum HI. Brain Tumor Stem Cells. Pediatr Res. 2006;59:54-8. https://doi. org/10.1203/01.pdr.0000203568.63482.f9

46. Thompson MC, Fuller C, Hogg TL, Dalton J, Finkelstein D, Lau CC, et al. Genomics identifies medulloblastoma subgroups that are enriched for specific genetic alterations. J Clin Oncol. 2006;24(12):1924-31. https://doi.org/10.1200/JCO.2005.04.4974

47. Kool M, Koster J, Bunt J, Hasselt NE, Lakeman A, van Sluis P, et al. Integrated Genomics Identifies Five Medulloblastoma Subtypes with Distinct Genetic Profiles, Pathway Signatures and Clinicopathological Features. PLOS ONE. 2008;3(8). https://doi.org/10.1371/journal.pone.0003088

48. Northcott P, Korshunov A, Witt H, Hielscher T, Eberhart CG, Mack S, et al. Medulloblastoma comprises four distinct molecular variants. J Clin Oncol. 2011;29(11):1408-14. https://doi.org/10.1200/ JCO.2009.27.4324

49. Cho Y-J, Tsherniak A, Tamayo P, Santagata S, Ligon A, Greulich H, et al. Integrative genomic analysis of medulloblastoma identifies a molecular subgroup that drives poor clinical outcome. J Clin Oncol. 2011;29(11):1424-30. https://doi.org/10.1200/JCO.2010.28.5148

50. Ahmad Z, Jasnos L, Gil V, Howell L, Hallsworth A, Petrie K, et al. Molecular and In Vivo Characterization of Cancer-Propagating Cells Derived from MYCN-Dependent Medulloblastoma. PLOS ONE. 2015. https://doi.org/10.1371/journal.pone.0119834 
51. Meco D, Servidei T, Lamorte G, Binda E, Arena V, Riccardi R. Ependymoma stem cells are highly sensitive to temozolomide in vitro and in orthopic models. Neuro-Oncology. 2014;16:1067-77. https://doi.org/10.1093/neuonc/nou008

52. Srikanthan D, Taccone MS, Van Ommeren R, Ishida J, Krumholtz SL, Rutka JT. Diffuse intrinsic pontine glioma: current insights and future directions. Chin Neurosurg J. 2021;7(1):6. https://doi. org/10.1186/s41016-020-00218-w

53. Xu C, Liu X, Geng Y, Bai Q, Pan C, Sun Y, et al. Patient-derived DIPG cells preserve stem-like characteristics and generate orthotopic tumors. Oncotarget. 2017;8(44):76644-55. https://doi.org/10.18632/ oncotarget. 19656

54. Wenger A, Larsson S, Danielsson A, Elbæk KJ, Kettunen P, Tisell M, et al. Stem cell cultures derived from pediatric brain tumors accurately model the originating tumors. Oncotarget. 2017;8:18626-39. https://doi.org/10.18632/oncotarget.14826

55. Bao S, Wu Q, Sathornsumetee S, Hao Y, Li Z, Hjelmeland AB, et al. Stem Cell-like Glioma Cells Promote Tumor Angiogenesis through Vascular Endothelial Growth Factor. Cancer Res. 2006;66:7843-8. https://doi.org/10.1158/0008-5472.CAN-06-1010

56. Karsy M, Albert L, Tobias ME, Murali R, Jhanwar-Uniyal M. All-trans retinoic acid modulates cancer stem cells of glioblastoma multiforme in an MAPK-dependent manner. Anticancer Res. 2010;30:4915-20.

57. Mostoufi-Moab S, Grimberg A. Pediatric Brain Tumor Treatment: Growth Consequences and their Management. Pediatr Endocrinol Rev. 2010;8(1):6-17.

58. Clayton PE, Shalet SM, Price DA. Growth response to growth hormone therapy following craniospinal irradiation. Eur J Pediatr. 1988;147(6):597-601. https://doi.org/10.1007/BF00442471

59. Ogilvy-Stuart AL, Shalet SM. Growth and puberty after growth hormone treatment after irradiation for brain tumours. Arch Dis Child. 1995;73(2):141-6. https://doi.org/10.1136/adc.73.2.141

60. Sulmont V, Brauner R, Fontoura M, Rappaport R. Response to growth hormone treatment and final height after cranial or craniospinal irradiation. Acta Paediatr Scand. 1990;79(5):542-9. https://doi. org/10.1111/j.1651-2227.1990.tb11509.x

61. Darendeliler F, Livesey EA, Hindmarsh PC, Brook CG. Growth and growth hormone secretion in children following treatment of brain tumours with radiotherapy. Acta Paediatr Scand. 1990;79(10): 950-6. https://doi.org/10.1111/j.1651-2227.1990.tb11357.x

62. Xu W, Janss A, Packer RJ, Phillips P, Goldwein J, Moshang T, Jr. Endocrine outcome in children with medulloblastoma treated with 18 Gy of craniospinal radiation therapy. Neuro-oncology. 2004;6(2):113-8. https://doi.org/10.1215/S1152851703000462

63. Jenkin D, Danjoux C, Greenberg M. Subsequent Quality of Life for Children Irradiated for a Brain Tumor Before Age Four Years. Med Pediatr Oncol. 1998;31:506-11. https://doi.org/10.1002/(SICI) 1096-911X(199812)31:6<506::AID-MPO7>3.0.CO;2-X

64. Mulhern RK, Horowitz ME, Kovnar EH, Langston J, Sanford RA, Kun LE. Neurodevelopmental Status of Infants and Young Children Treated for Brain Tumors With Preirradiation Chemotherapy. J Clin Oncol. 1989;7(11):1660-6. https://doi.org/10.1200/JCO.1989.7.11.1660

65. Nimmervoll BV, Boulos N, Bianski B, Dapper J, DeCuypere M, Shelat A, et al. Establishing a Preclinical Multidisciplinary Board for Brain Tumors. Clin Cancer Res. 2018;24(7):1654-66. https://doi. org/10.1158/1078-0432.CCR-17-2168

66. Aldape K, Brindle KM, Chesler L, Chopra R, Gajjar A, Gilbert MR, et al. Challenges to curing primary brain tumours. Clin Oncol. 2019;16:509-20. https://doi.org/10.1038/s41571-019-0177-5 